\title{
EVERY CURVE ON A NONSINGULAR SURFACE CAN BE DEFINED BY TWO EQUATIONS
}

\author{
M. BORATYŃSKI
}

\begin{abstract}
Let $R$ be a smooth two-dimensional affine algebra over an algebraically closed field, and let $I$ be an unmixed ideal of height one in $R$. Then there exist $a, b$ in $I$ such that $\operatorname{rad}(I)=\operatorname{rad}(a, b)$.
\end{abstract}

Van der Waerden [6] has proved that every subvariety of an $n$-dimensional affine variety can be defined by $n+1$ equations. Easy examples (for instance a nontorsion point on a nonsingular affine curve) show that this is, in general, the best possible result.

The aim of this note is to prove the statement of the title, which is an improvement of van der Waerden's theorem in a somewhat special case. Its algebraic version, which we are going to prove, runs as follows:

THEOREM. Let $R$ be a smooth two-dimensional affine algebra over an algebraically closed field, and let $I$ be an unmixed ideal of height 1 of $R$. Then there exists $a, b \in I$ such that $\operatorname{rad} I=\operatorname{rad}(a, b)$.

1. Preliminaries. In this section we briefly state some basic results about Chern classes that we will use in what follows. For the details we refer to [3].

Let $X$ be a smooth variety over an algebraically closed field, and let $A(X)=$ $\bigoplus_{i=0}^{\operatorname{dim} X} A^{i}(X)$ denote the Chow ring of $X . A^{i}(X)$ consists of rational equivalence classes of codimension- $i$ cycles of $X$.

For any $f: X \rightarrow Y$ ( $Y$ smooth), one has the induced ring homomorphism $f^{*}: A(Y) \rightarrow A(X)$. In particular, for any open subvariety $U \subset X$, there is an induced map $i^{*}: A(X) \rightarrow A(U)$, where $i$ denotes the inclusion. The kernel of $i^{*}$ is generated by the cycles with support contained in $X-U[2]$.

For any locally free sheaf $\mp$ over $X$, one defines, for $i \geq 0$, the elements $c_{i}(\mp) \in$ $A^{i}(X)$ such that $c_{0}(\mathcal{F})=1$ and $c_{i}(\mathcal{F})=0$ for $i>\operatorname{rk} \mathcal{F}$. If $\mathrm{rk} \mp=1$, then $c_{1}(\mathcal{F})$ is equal to the image of $\mathcal{F}$ in $A^{1}(X)=$ Pic $X$. In general, $c_{1}(\mathcal{F})=c_{1}\left(\bigwedge^{\text {rank }} \mathcal{F}\right)$. Let $0 \rightarrow \mathcal{F}^{\prime} \rightarrow \mathcal{F} \rightarrow \mathcal{F}^{\prime \prime} \rightarrow 0$ be an exact sequence of locally free sheaves over $X$. Then

$$
c_{k}(\xi)=\sum_{i+j=k} c_{i}\left(\mathcal{F}^{\prime}\right) c_{j}\left(\mathcal{F}^{\prime \prime}\right) .
$$

In particular, $c_{i}(\mathcal{F})=0$, for $i>0$, if $\mathcal{F}$ is free.

Let $₹$ be a locally free sheaf of rank $k$. Suppose there exists a section of $₹$ whose scheme of zeros is of codimension $k$. Then $c_{k}(\mathcal{F})$ is represented by the corresponding cycle of zeros.

Let $R$ be a smooth affine algebra over an algebraically closed field, and let $X$ denote the corresponding affine (smooth) variety. Then for any projective $R$-module

Received by the editors August 1, 1983.

1980 Mathematics Subject Classification. Primary 13C05, 14C99, $14 \mathrm{M} 07$. 
$P$, one defines, for $i \geq 0, c_{i}(P)=c_{i}(\tilde{P}) \in A^{i}(X)$, where $\tilde{P}$ is the corresponding locally free sheaf over $X$.

Let $P$ and $I$ be the projective $R$-modules of ranks 2 and 1, respectively. In what follows we shall need the following formulas:

$$
\begin{aligned}
& c_{1}(P \otimes I)=c_{1}(P)+2 c_{1}(I), \\
& c_{2}(P \otimes I)=c_{2}(P)+c_{1}(P) c_{1}(I)+c_{1}^{2}(I) .
\end{aligned}
$$

One can easily obtain them by using the "splitting principle".

For the sake of completeness, following $[\mathbf{1}]$, we shall recall briefly the definition of $A^{i}(X)$ in the affine case. Let $R$ denote the coordinate ring of $X$, and let $F_{i}$ stand for the free group generated by the prime ideals of $R$ of ht $i$. For an equicodimensional ideal $I$ of $R$ with ht $I=i$, we let $[I]=\sum \operatorname{length}\left(R_{p} / I_{p}\right) p \in F_{i}$, with the summation ranging over all minimal primes of $I$. By definition $A^{i}(X)=F_{i} / G_{i}$, where $G_{i}$ denotes the subgroup of $F_{i}$ generated by $[(q, f)]$ with $q$ prime of height $i-1$ and $f \in R \backslash q$.

2. Proof of the Theorem. Let $X=\operatorname{Spec} R$ and $U=\operatorname{Spec} R-V(I)$. $I$ is a projective $R$-module, since $R$ is regular. $c_{1}^{2}(I) \in A^{2}(X)$ and maps to zero in $A^{2}(U)$. Therefore, $c_{1}^{2}(I)$ can be represented by the cycle $\sum_{i=1}^{t} k_{i} \mathfrak{m}_{i}$, with $\mathfrak{m}_{i}$ being the maximal ideals of $R$ containing $I$. Suppose that $k_{i}<0$ for $1 \leq i \leq s$ and $k_{i} \geq 0$ for $s<i \leq t$. Let $q_{i}$ be a minimal prime of $I$ contained in $\mathfrak{m}_{i}$ for $i=$ $1, \ldots, s$. Then, for $f_{i} \notin q_{i}$ contained in sufficiently high powers of $\mathfrak{m}_{i}$ for $1 \leq i \leq s$, $\sum_{i=1}^{t} k_{i} \mathrm{~m}_{i}+\sum_{i=1}^{s}\left[\left(q_{i}, f_{i}\right)\right]$ is an effective cycle. So we can assume that $c_{1}^{2}(I)$ is represented by $\sum_{i=1}^{t} k_{i} \mathfrak{m}_{i}$ with $k_{i} \geq 0$. Let $x_{i}, y_{i} \in \mathfrak{m}_{i}$ generate $\left(\mathfrak{m}_{i}\right)_{\mathfrak{m}_{i}}$, and let $J_{i}$ denote the $\mathfrak{m}_{i}$-primary component of $\left(x_{i}, y_{i}^{k_{i}}\right)$. Then $[J]=\sum_{i=1}^{t} k_{i} \mathfrak{m}_{i}=c_{1}^{2}(I)$, where $J=\prod_{i=1}^{t} J_{i} . J$ is a locally complete intersection height 2 ideal of $R$. So by [5] there exists an exact sequence $0 \rightarrow R \rightarrow P \rightarrow J \rightarrow 0$ with $P$ projective of rank 2. We have $c_{1}(P)=0$, since $\Lambda^{2} P \simeq R$ and $c_{2}(P)=[J]=c_{1}^{2}(I)$. After tensoring the above exact sequence with $I$, we get the exact sequence

$$
0 \rightarrow I \rightarrow P \otimes I \rightarrow J \otimes I=J I \rightarrow 0 .
$$

$I / J I \simeq I^{-1} / J I^{-1}$, since both modules are projective of rank 1 over $R / J$, which is a 0 -dimensional ring.

Let $\varphi: I \rightarrow I^{-1}$ be a lifting of this isomorphism, and let

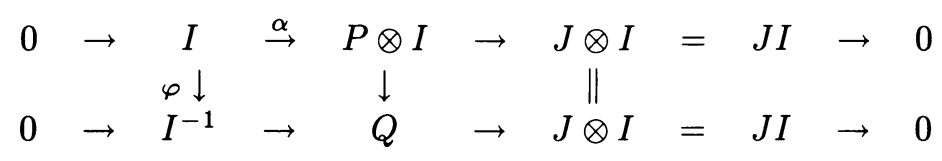

be the corresponding push-out diagram. From the definition of "push-out" we get the exact sequence

$$
0 \rightarrow I \rightarrow I^{-1} \oplus(P \otimes I) \rightarrow Q \rightarrow 0 .
$$

$Q$ is a projective $R$-module of rank 2 , since $\alpha$ is locally a homomorphism $R \stackrel{[a, b]}{\rightarrow} R^{2}$, with $(a, b)=J$, and $\varphi$ is locally a homomorphism $R \stackrel{c}{\rightarrow} R$, with $c$ comaximal to $J$.

Using the multiplicative property of Chern classes, the formula for the Chern classes of $P \otimes I$, and the facts that $c_{1}(P)=0$ and $c_{2}(P)=c_{1}^{2}(I)$, one obtains, after tedious calculations, that $c_{1}(Q)=0$ and $c_{2}(Q)=0$. It follows from [4] that $Q$ is 
free. Thus, $J I$ is generated by two elements. $\operatorname{rad} I=\operatorname{rad} J I$ since the ideals $\mathfrak{m}_{i}$ contain $I$. Thus, there exist $a, b \in I$ such that $\operatorname{rad} I=\operatorname{rad}(a, b)$ which completes the proof of the Theorem.

REMARKS. 1. Every curve on a nonsingular surface can be defined by one equation if and only if its class group is torsion.

2. One can wonder about possible generalization of the Theorem to a higherdimensional case. In particular, one can ask for which numbers $d, n$, with $d<n$, can every (pure) $d$-codimensional Cohen-Macaulay subvariety of an $n$-dimensional affine variety (over an algebraically closed field) be defined by $n$ equations.

ADDED IN PROOF. After this paper was written N. Mohan Kumar showed the following:

1. The Theorem holds true without the smoothness assumption on $R$ provided $I$ is locally principal. One can easily deduce from this that every locally complete intersection curve in an affine $n$-fold over an algebraically closed field is set-theoretically defined by $n$ equations.

2. Every divisor on a smooth 3-fold over an algebraically closed field is set-theoretically defined by 3 equations.

Both these results were generalized by M. P. Murthy who proved that every locally principal ideal of a regular $n$-dimensional affine algebra $R$ over an algebraically closed field is generated up to radical by $n$ elements provided $n=\operatorname{dim} R \geq 2$. G. Lyubeznik has proved that every locally complete intersection ideal of an $n$ dimensional CM ring $R$ is generated up to radical by $n$ elements if $1<$ ht $I<n$.

In the meantime the author was able to prove the following: Let $I$ be a locally principal ideal of a two-dimensional affine algebra $R$ over an algebraically closed field. Then the ideal $(I, x)$ with $x \in R$ is generated up to radical by two elements provided $(I, x)=J \cdot K$ with $J$-locally principal and $K=R$ or ht $K=2$. That assumption holds automatically if $x \in I$ or if $R$ is regular. This suggests that some of the above-mentioned results may carry over to certain ideals which are not necessarily unmixed.

\section{REFERENCES}

1. L. Claborn and R. Fossum, Generalization of the notion of class group, Illinois J. Math. 12 (1968), 228-253.

2. W. Fulton, Rational equivalence on singular varieties, Inst. Hautes Études Sci. Publ. Math. 45 (1975), 147-167.

3. A. Grothendieck, La théorie des classes de Chern, Bull. Soc. Math. France 86 (1958), 137-154.

4. M. P. Murthy and R. G. Swan, Vector bundles over affine surfaces, Invent. Math. 36 (1976), 125-165.

5. J. P. Serre, Sur les modules projectives, Sem. Dubreil-Pisot, 1960/1961.

6. B. L. van der Waerden, Review, Zbl. Math. 24 (1941), 276.

Institute of Mathematics, Polish ACAdemy of SCiences, P. O. BoX 137, 00-950 WARSAW, ŚNIADECKICH 8, POLAND

Current address: Department of Mathematics, SUNY at Albany, Albany, New York 12222 